\title{
Epidémiologie des nématodes gastro-intestinaux chez les petits ruminants dans la région des plateaux au Togo
}

\author{
B. Bonfoh ${ }^{1 *}$, J. Zinsstag ${ }^{2}$, P. Ankers ${ }^{1}$, L.J. Pangui ${ }^{3}$, K. Pfister ${ }^{4}$
}

BONFOH B., Zinsstag J., ANKers P., PANGUi L.J., PFister K. Epidémiologie des nématodes gastro-intestinaux chez les petits ruminants dans la région des plateaux au Togo. Revue Elev. Méd. vét. Pays trop., 1995, 48 (4) : 321-326

Des autopsies helminthologiques ont été effectuées au Togo sur des petits ruminants ( 59 ovins, 60 caprins), de race Djallonké, révélant la présence de huit espèces de nématodes gastro-intestinaux qui sont par ordre de prévalence Trichostrongylus sp. (T. axei et $T$. colubriformis) (99 p. 100), Haemonchus contortus (82 p. 100), Strongyloides papillosus (67 p. 100), Cooperia curticei (43 p. 100), Oesophagostomum columbianum (40 p. 100), Gaigeria pachyscelis (36 p. 100) et $T r i$ churis ovis (4 p. 100). L'intensité de la communauté parasitaire est de $1367 \pm 146$ chez les ovins et de $1133 \pm 102$ chez les caprins avec une communauté adulte qui culmine en août-septembre (moutons : 2135 \pm 494 , chèvres : $2066 \pm 270$ ). En saison sèche, $H$. contortus et $O$ columbianum entrent en hypobiose larvaire (L4). Le nombre d'ceufs par gramme de matière fécale est en général élevé $(>3000)$ et ni l'espèce ni l'âge de l'hôte (6 mois à 3 ans) n'a d'effet significatif sur la charge parasitaire.

Mots-clés : Ovin - Caprin - Strongylidae - Nematoda - Infestation - Helminthologie - Epidémiologie - Saison - Togo.

\section{INTRODUCTION}

Les races de petits ruminants rencontrées au Togo sont les races Djallonké, Sahélienne ainsi que le mouton de Vogan (croisement d'absorption du mouton Djallonké par le mouton Sahélien). L'amélioration de leur élevage y est soutenue à travers le Projet national petits ruminants. Mais la grande majorité des 2077000 petits ruminants du Togo est encore élevée de façon entièrement traditionnelle. La divagation reste le mode d'élevage le plus répandu sauf pendant la période des cultures et récoltes

1. Projet helminthoses de I'Université de Neuchâtel, Centre International de Trypanotolérance, PMB 14, Banjul, Gambie.

2. Centre Suisse de Recherches Scientifiques (C.S.R.S.), BP 1303, Abidjan 01, Côte d'Ivoire.

3. Ecole Inter-Etats des Sciences et Médecine Vétérinaires (E.I.S.M.V.), BP 5077, Dakar Hann, Sénégal.

4. Labo. Pfister, Morgenstr 83B, CH-3018 Berne, Suisse.

* Pour la correspondance : Projet Gambie, Vétérinaires Sans Frontières-Suisse (VSF-CH), PMB 107, Banjul, Gambie.

Reçu le 29.11.94, accepté le 19.2.96. où les animaux sont en enclos ou pâturent sous surveillance des enfants. L'alimentation se compose de toute végétation accessible, de sous-produits agricoles et de restes de cuisine. Ces petits ruminants, qui bénéficient rarement de soins vétérinaires, sont soumis à de fortes contraintes pathologiques, notamment la peste des petits ruminants, la pasteurellose et les nématodoses gastro-intestinales (Sant'Anna, 1989 ; Adomefa, 1990 ; résultats non publiés).

Le présent article décrit les résultats d'une série d'autopsies de petits ruminants ayant permis de faire l'inventaire des nématodes gastro-intestinaux, de définir leur prévalence et leur charge parasitaire dans la région des plateaux au Togo. La conduite des autopsies sur dix mois a aussi permis de définir l'influence des saisons en plus de celle de l'âge de l'hôte sur la charge parasitaire.

Ces résultats ont été ensuite utilisés pour élaborer un calendrier de vermifugation spécifique à cette zone climatique.

\section{MATERIEL et METHODES}

\section{Période et zone d'étude}

L'expérience s'est déroulée de janvier à octobre 1992 dans la préfecture de Kloto (région des plateaux) à 120 $\mathrm{km}$ de Lomé. Cette région, d'une altitude variant entre 300 et 800 mètres, bénéficie d'un climat subéquatorial avec une pluviométrie moyenne de $1500 \mathrm{~mm} / \mathrm{an}$ tombant entre mars et octobre. La moyenne des températures minimales et maximales est respectivement de $25^{\circ} \mathrm{C}$ et $29^{\circ} \mathrm{C}$.

\section{Les animaux}

Les tractus digestifs de 60 caprins et 59 ovins, achetés à l'abattoir municipal de Kpalimé (chef lieu de la préfecture de Kloto), ont été lavés à raison de trois par semaine. Ces animaux, âgés entre six mois et trois ans, étaient tous de race Djallonké et provenaient d'élevages traditionnels de la région des plateaux. De janvier à octobre 1992, quatre à huit animaux par mois étaient prélevés par espèce animale. 


\section{B. Bonfoh J. Zinsstag P. Ankers L.J. Pangui K. Pfister}

\section{Examens parasitologiques}

Des échantillons de fèces ont été prélevés pour la coprologie quantitative par la technique de McMaster (6) et qualitative par les méthodes de flottation et sédimentation (11). Après l'abattage, la recherche des nématodes gastro-intestinaux s'est faite selon la méthode décrite par Graber et Perrotin (7). Le tractus digestif est ligaturé en trois points afin d'isoler l'abomasum, l'intestin grêle et le gros intestin. Chacune de ces portions est lavée séparément afin de récupérer les nématodes gastro-intestinaux. Les parasites se trouvant dans une partie aliquote représentant $1 / 15$ e du contenu total de chaque portion mis en suspension homogène sont comptés et identifiés après éclaircissement à l'acide lactique 90 p. 100. La moitié de la muqueuse de l'abomasum est digérée pour y compter les larves présentes (7).

\section{Analyse statistique}

Un test non paramétrique (Wilcoxon: somme des rangs) a été utilisé pour déterminer l'influence des différents facteurs sur les charges parasitaires.

\section{RESULTATS}

\section{Examens coproscopiques (figure 1)}

L'excrétion moyenne d'œufs de nématodes gastro-intestinaux sur l'ensemble des animaux autopsiés (exprimée en œufs par gramme de fèces ou OPG) a été de $7812 \pm$ 874 pour les œufs de strongles et de $1033 \pm 162$ pour

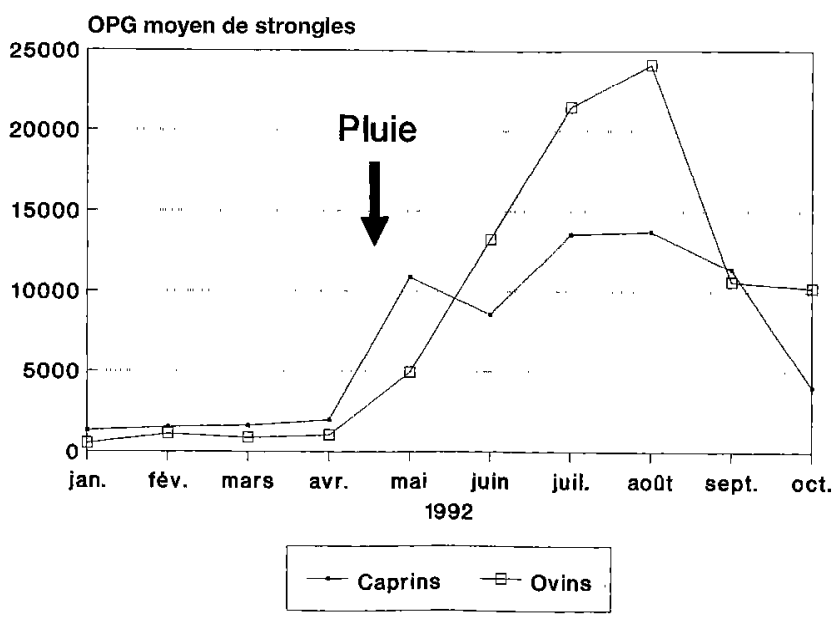

Figure 1 : Fluctuation saisonnière de l'excrétion d'wufs de strongles (œufs par gramme de matières fécales) chez les petits ruminants de la région des plateaux au Togo. les œufs de Strongyloides spp. Cette excrétion culmine en août et est à son plus bas niveau entre janvier et avril chez les moutons comme chez les chèvres. Seul un mouton excrétait 1100 OPG de Trichuris spp.

\section{Autopsies helminthologiques}

Tous les petits ruminants autopsiés étaient infestés. Les charges ainsi que les prévalences de parasites identifiés sont décrits dans le tableau I avec comme parasite le plus important, en ce qui concerne l'intensité et la prévalence chez les deux espèces hôtes confondues, Trichostrongylus sp. (99 p. 100), suivi de Haemonchus contortus (82 p. 100), Strongyloides papillosus (67 p. 100) et Cooperia curticei (43 p. 100).

Sur l'ensemble des petits ruminants, les classes d'âge 6 mois, 1,2, et 3 ans ont des charges de parasites adultes respectives de $1310,1133,1143$ et 1064 nématodes. II n'y a pas de différence significative de la charge parasitaire entre ovins et caprins (tableau II).

\section{Variations saisonnières des charges parasitaires}

La charge parasitaire et la fluctuation de la communauté de nématodes sont similaires chez les caprins et chez les ovins (figure 2) avec une moyenne de $722 \pm 78$ (janvier à avril) et $1605 \pm 123$ (mai à octobre) pour l'ensemble des animaux.

La charge parasitaire des populations juvéniles et adultes d'H. contortus juvéniles et adultes (figure 3 ) est influen-

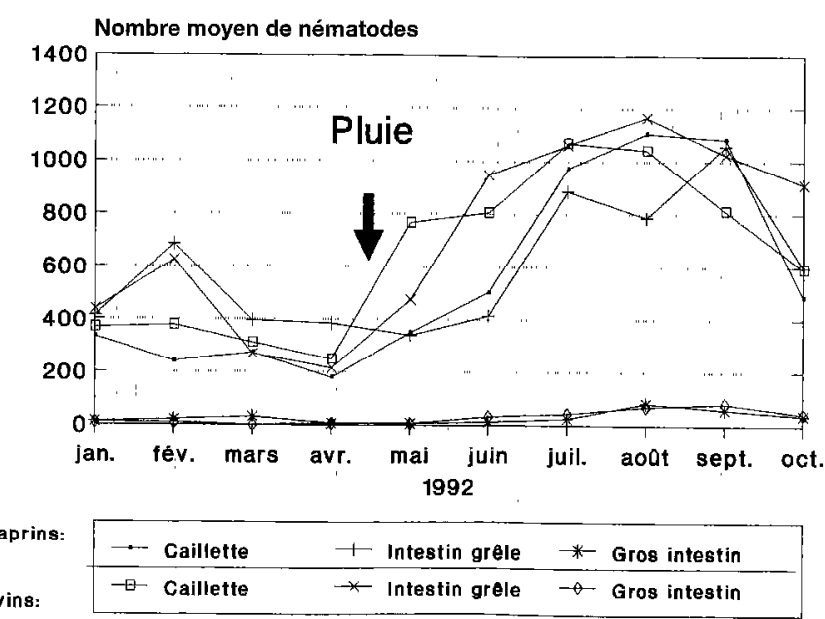

Figure 2 : Fluctuations saisonnières des populations de nématodes gustro-intestinaux (valeurs moyennes) chez les petits ruminants de la région des plateaux au Togo. 
Prévalence et intensité de la faune parasitaire rencontrée chez 119 petits ruminants autopsiés provenant de la région des plateaux au Togo

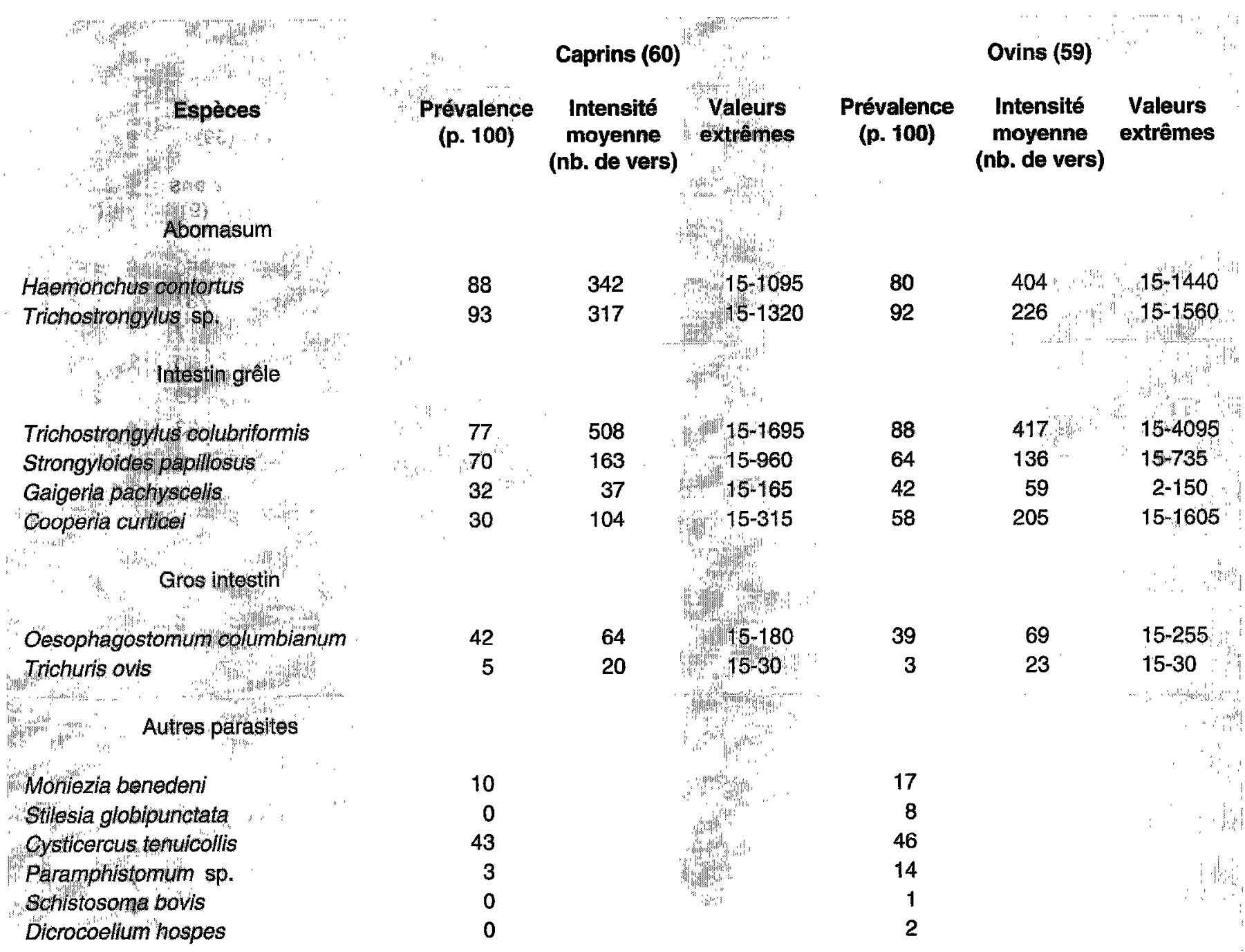

cée de façon significative $(P<0,005)$ par les saisons alors que ni l'âge, ni l'espèce animale n'ont d'effet significatif sur ces populations. Dans l'abomasum, la charge parasitaire de la population d' $H$. contortus (adultes et juvéniles) est élevée pendant les mois de juillet et août $(688 \pm 69)$. La population larvaire (L4) moyenne est faible durant cette même période $(6 \pm 2)$ mais est maximale entre mars et mai $(136 \pm 15)$.

Dans l'intestin grêle, Trichostrongylus sp. est présent durant toute la période d'étude. II est à l'origine d'un pre- mier pic de la population parasitaire en février (figure 2) avec une abondance de $614 \pm 108$ puis il culmine entre mai et septembre à $923 \pm 97$. L'espèce de l'hôte n'a pas d'influence significative.

Trichostrongylus colubriformis cohabite dans la caillette avec Trichostrongylus axei chez 34 p. 100 des petits ruminants autopsiés. Les populations adultes de $S$. papillosus, C. curticei et $G$. pachyscelis restent relativement stables pendant toute l'année avec toutefois une légère baisse de janvier à mai. 
政

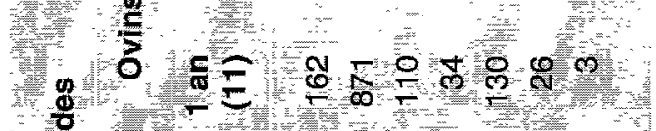
tan

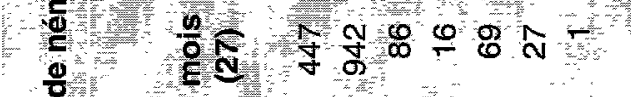
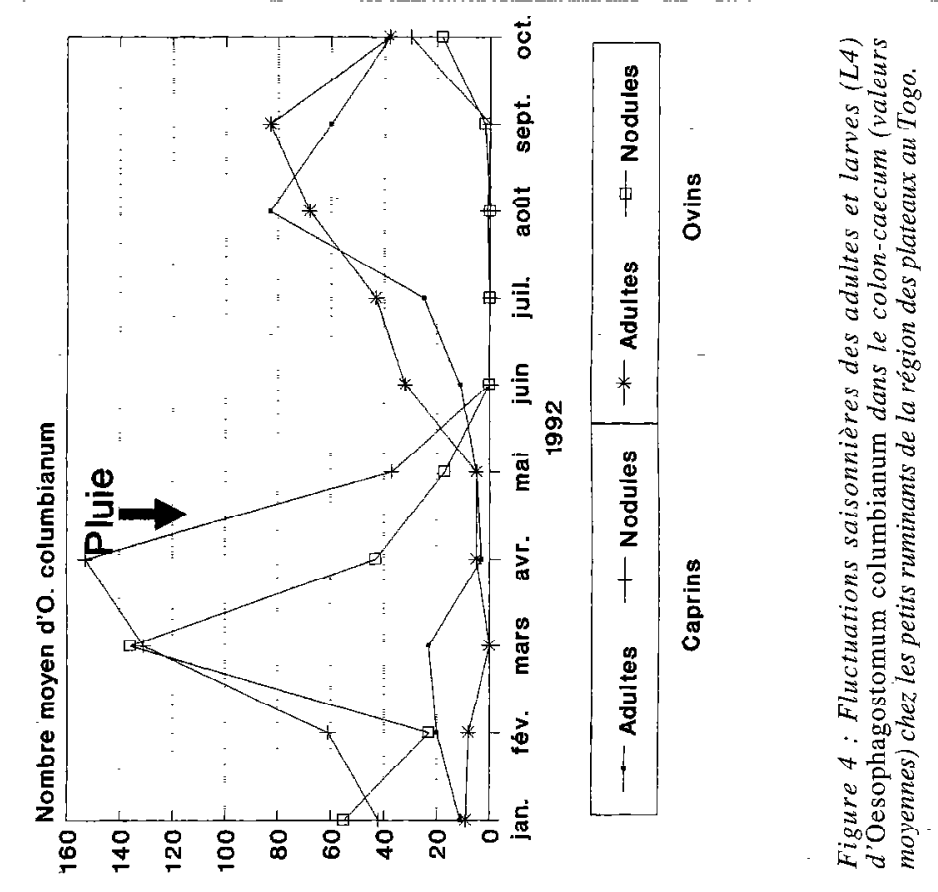
อ

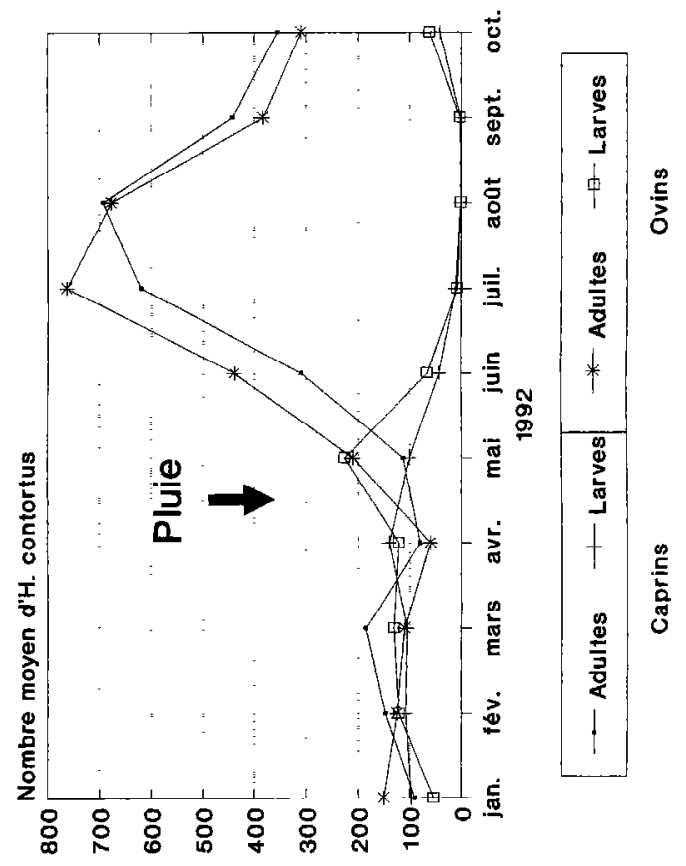


Dans le gros intestin (figure 4) on note un nombre élevé de nodules inflammatoires d' $O$. columbianum sur le colon-caecum en mars-avril $(118 \pm 27)$. On a constaté une augmentation de la charge parasitaire de juvéniles et d'adultes en août et septembre $(74 \pm 18$ ) sans pour autant que cette variation ne soit significative. Seuls 5 animaux étaient porteurs de $T$. ovis adultes avec une intensité moyenne de $21 \pm 4$

\section{DISCUSSION}

Les valeurs coproscopiques d'œufs de strongles gastrointestinaux sont, chez les petits ruminants, significativement plus élevées en période de pluies qu'en période sèche $(P<0,001)$ où l'on remarque le phénomène d'hypobiose avec la présence de larves quiescentes. Mais il n'y a pas d'élévation de l'OPG précédant les premières pluies telle qu'elle est décrite en Gambie $(1,5)$.

La totalité des animaux autopsiés portent au moins l'un des nématodes mentionnés dans le tableau I. Les variations de charges entre les ovins et les caprins ne sont pas significatives. Par contre il a été rapporté au Sénégal de fortes prévalences des helminthes chez les ovins comparés aux caprins (9).

Les parasites recensés sont les mêmes que ceux rencontrés entre autres au Tchad (7), au Nigéria (4), au Sénégal $(7,9,13)$ et en Gambie, pays avec un climat soudano-sahélien (5).

$H$. contortus et Trichostrongylus sp. sont les parasites dominants chez les petits ruminants au Togo. La population d' $H$. contortus survit pendant la saison sèche en partie sous forme de larves hypobiotiques (L4) dans la muqueuse de la caillette. Ce phénomène lié aux facteurs écologiques (2) est rapporté plus au nord, entre autres au Sénégal $(8,12)$ et en Gambie (5). Cette inhibition au stade larvaire en saison sèche se voit aussi chez $O$. columbianum où la saison joue un rôle significatif sur l'évolution du nombre de nodules $(P<0,001)$ mais pas sur la population adulte. Cela s'avère d'autant plus important que l'œsophagostomose est due essentiellement à la présence d'un nombre élevé de nodules et de larves sur le gros intestin.

L'importance de ces nodules en saison sèche a été rapportée au Sénégal $(3,11)$. Trichostrongylus $\mathrm{sp}$. sévit durant toute l'année sous forme adulte avec une population et une prévalence élevées. Il représente le nématode dominant dans la région des plateaux et a été signalé comme pouvant provoquer des pertes de poids importantes chez les ovins (10).

Les autres nématodes (Strongyloides papillosus, Coope ria curticei, Gaigeria pachyscelis et Trichuris ovis) ont été trouvés avec des intensités faibles. Mais une importance particulière peut être accordée à $G$. pachyscelis qui sévit pendant toute l'année et qui est pathogène à de faibles intensités (7).

L'absence d'effet de l'âge de l'hôte sur la communauté parasitaire a aussi été relevé par exemple au Tchad (7) et en Gambie (5).

\section{CONCLUSION}

Sur la base de ces résultats, nous suggérons de faire deux traitements par an : une vermifugation en juin contrôlant la population adulte déjà importante et un deuxième traitement en octobre afin d'éliminer les nouvelles populations adultes et les larves déjà en hypobiose.

Seule une étude économique exhaustive permettrait de déterminer les bénéfices éventuels qu'apporterait ce schéma de vermifugation des animaux cibles.

\section{Remerciements}

Les auteurs tiennent à remercier la Direction de la coopération au développement et de l'aide humanitaire suisse (DDA) qui a financé ce travail à travers le Projet helminthoses de l'Université de Neuchâtel (UNP). Leurs remerciements vont également au Professeur Leo Dempfle et au Docteur Bakary Touray, Directeurs du Centre international de trypanotolérance ainsi qu'au Docteur Ignace Kombaté, Directeur de l'Institut national zootechnique et vétérinaire au Togo pour avoir mis à leur disposition les infrastructures nécessaires à la présente étude. Ils remercient enfin l'équipe du projet UNP en Gambie et les bouchers de Kpalimé.

\section{Bibliographie}

1. ANKERS P., ZINSSTAG J., PFISTER K., 1994. Quasi-absence de réinfestation par les strongles du bétail gambien en saison sèche. Revue Elev. Méd. vét. Pavs trop. 47 (2): $201-205$.

2. ARMOUR J.. 1980. The epidemiology of helminth diseases in farm animals. Vet. Parasitol, 6: 7-46.

3. BELOT J., PANGUI L.J., 1986. Observations sur la fertilité des strongles digestifs du mouton dans le cadre d'une étude ponctuelle aux abattoirs de Dakar : remarques préliminaires et nodules parasitaires. Revue Méd. vét. 137 : 533-536.

4. FABIYI J.P. OPEMAN D.B., HUTCHINSON G.W., 1989. Abundance and survival of infective larvae of the cattle nematodes Cooperia punctata, Haenonchus placei and Oesophagostomum radiatum from feacal pats in a wet tropical climate. Aust. Vet., 65 (8): 229-231. 
5. FRITSCHE T., KAUFMANN J., PFISTER K., 1993. Parasite spectrum and seasonal epidemiology of gastrointestinal nematodes of small ruminants in the Gambia. Vet. Parasitol., 49: 271-283.

6. GORDON H.Mch., WHITLOCK H.V., 1939. A new technique for counting nematode eggs in sheep faeces. J. Conc. Sci. Ind. Res. Aust., $12: 50-$ 52.

7. GRABER M., PERROTIN G., 1983. IIelminthes et helminthoses de ruminants domestiques d'Afrique tropicale. Paris, France, Editions du Point vétérinaire, $162 \mathrm{p}$.

8. NDAO M., 1991. Contribution à l'étude de l'épidémiologie des nématodes gastro-intestinaux des ruminants dans la zone sylvo-pastorale du Sénégal. Thèse en Médecine vétérinaire, Université de Dakar, Dakar, Sénégal. $\left(n^{\circ} 35\right)$

BONFOH B., ZINSSTAG J., ANKERS P., PANGUI L.J., PFISTER K. Epidemiology of gastrointestinal nematodes in small ruminants in the "région des plateaux" in Togo. Revue Elev. Méd. vét. Pays trop., 1995, 48 (4) : $321-326$

Post-mortem helminthological examinations were performed in Togo on small ruminants of the Djallonké breed ( 59 sheep, 60 goats), revealing the presence of eight gastrointestinal nematode species which were, in decreasing prevalence order, Trichostrongylus sp. ( $T$. axei and T. colubriformis) (99\%), Haemonchus contortus $(82 \%)$, Strongyloides papillosus $(67 \%)$, Cooperia curticei $(43 \%)$, Oesophagostomum columbianum $(40 \%)$, Gaigeria pachyscelis (36 \%) and Trichuris ovis $(4 \%)$. The average worm burden was $1367 \pm 146$ in sheep and $1133 \pm 102$ in goats with an adult worm population peak in August-September (sheep: $2135 \pm 494$, goats: $2066 \pm 270$ ). During the dry season, Haemonchus contortus and Oesophagostomum columbianum populations were mainly in larval hypobiosis $(L 4)$. The number of eggs per gram faeces was usually high $(>3000)$ and neither the species nor the age of the host ( 6 months to 3 years) played a significant role in helminth abundance.

Key words: Sheep - Goat - Strongylidae - Nematoda - Infestation - Helminthology - Epidemiology - Season - Togo.
9. NDAO M., BELOT J., ZINSSTAG J., PTISTER K., 1995. Epidémiologie des helminthoses gastro-intestinales des petits ruminants dans la zone sylvo-pastorale au Sénégal. Vet. Res., 26 : 132-139.

10. SHUMARD R.F., BOLIN D.W., EVELETH D.F., 1957. Physiological and nutritional changes in lambs infected with the nematodes, Haemonchus contortus, Trichostrongylus coluhriformis and Nematodirus spathiger. Am. J. Vet. Res., 18 : 330-337.

11. VASSILIADES G., 1981. Parasitisme gastro-intestinal chez le mouton du Sénégal. Revue Elev. Méd. vét. Pays trop., 34 : 169-177.

12. VERCRUYSSE J., 1983. The seasonal prevalence of inhibited development of Haemonchus contortus in sheep in Senegal. Vet. Parasitol., 17: $159-163$.

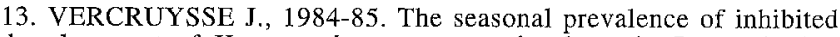
development of Haemonchus contortus in sheep in Senegal. Vet. Parasitol, 17: 159-163.

BONFOH B., ZINSSTAG J., ANKERS P., PANGUI L.J, PFISTER K. Epidemiología de los nemátodos gastroinstestinales en los pequeños rumiantes de la región de las mesetas en Togo. Revue Elev. Méd. vét. Pays trop., 1995, 48 (4) : 321-326

Se han efectuado en Togo, autopsias helpintológicas sobre pequeños rumiantes (59 ovinos, 60 caprinos), todos de raza Djallonke. Revelaron la presencia de ocho especies de nemátodos gastrointestinales que son, por orden de prevalencia, Trichostrongylus sp. (T. axei y T. colubriformis) ( 99 p. 100), Haemonchus contortus ( 82 p. 100), Strongyloides papillosus (67 p. 100), Cooperia curticei (43 p. 100), Oesophagostomum columbianum $(40$ p. 100), Gaigeria pachyscelis $(36$ p. 100) y Trichuris ovis (4 p. 100). Las cargas totales medias de vermes' en los ovinos y los caprinos son respectivemente $1367 \pm 146,1133 \pm 102$ con una población verminosa adulta total que culmina en agosto-septiembre (ovinos : $2135 \pm 494$, caprinos : $2066 \pm 270$ ). Durante la estación seca Haemonchus contortus y Oesophagostomum columbianum se quedan en hypobiosa larval. En general, el número de huevos por gramo de heces es elevado $(>\mathbf{3 0 0 0})$ y ni la especic ni la cdad del huésped (de 6 meses hasta 3 años) de los pequeños rumiantes no desempeña un papel significativo sobre la carga parasitaria.

Palabras clave : Ovino - Caprino - Infestacíon - Strongylidae - Nemato$d a$ - Helmintología - Epidemiología - Estacíon del años - Togo. 